SUNY-NTG-02/30

\title{
Replica Limit of the Toda Lattice Equation
}

\author{
K. Splittorff and J.J.M. Verbaarschot \\ Department of Physics and Astronomy, SUNY, Stony Brook, New York 11794
}

\begin{abstract}
In a recent breakthrough Kanzieper showed that it is possible to obtain exact nonperturbative Random Matrix results from the replica limit of the corresponding Painlevé equation. In this article we analyze the replica limit of the Toda lattice equation and obtain exact expressions for the two point function of the Gaussian Unitary Ensemble and the resolvent of the chiral Unitary Ensemble. In the latter case both the fully quenched and the partially quenched limit are considered. This derivation explains in a natural way the appearance of both compact and noncompact integrals, the hallmark of the supersymmetric method, in the replica limit of the expression for the resolvent. We also show that the supersymmetric partition function and the partition function with fermionic replicas are related through the Toda lattice equation.
\end{abstract}

Introduction. The replica trick originally introduced in [1] has been widely used in the theory of disordered systems ranging from spin glasses [1, 2, 3] to QCD 《4]. The quest for its rigorous understanding has been ongoing [5, 6, 6, 8, 9, 10, 11, 12, 13, 14, 15 and will be further pursued in this letter. The idea is to simplify the calculation of the disorder average of the logarithm of the partition function starting from

$$
\langle\log Z\rangle=\lim _{n \rightarrow 0}\left\langle\frac{Z^{n}-1}{n}\right\rangle .
$$

This method should work if we know the right hand side of this equation as an analytical function of $n$. In practice, though, one is usually only able to calculate $\left\langle Z^{n}\right\rangle$ for positive integer values of $n$ which is not sufficient to reproduce the complete function. For example, terms of the form $(\sin \pi n x) / n$ or singularities along the real $n$ axis could lead to a breakdown of the replica limit. The first problems with the replica trick appeared in the theory of spin glasses [2] where the result for the entropy turned out to be not positive definite. These problems were resolved by means of an intricate scheme of replica symmetry breaking [3]. Although the replica trick has been used widely in the theory of disordered systems, it has been criticized as well [5,8]. In particular, it has been a long standing belief that the replica trick can only be applied to asymptotic expansions which can be resummed in some cases [13]. This belief was shattered in recent breakthrough by Kanzieper 14. In this letter we will obtain further exact nonperturbative results from the replica limit of the Toda lattice equation. Both this method and the method of [14 rely on the relations between Random Matrix Theories and the theory of exactly integrable systems.

We will analyze the replica limit of correlation functions of a Random Matrix partition function. In the case of the resolvent we start from the identity

$$
G(x)=\frac{1}{N}\left\langle\operatorname{Tr} \frac{1}{x+D}\right\rangle=\frac{1}{N} \lim _{n \rightarrow 0} \partial_{x}\left\langle\frac{\operatorname{det}^{n}(D+x)}{n}\right\rangle .
$$

Here, $D$ is an $N \times N$ random matrix and the average is over the probability distribution of its matrix elements. The replica limit can be calculated in two different ways, by calculating the partition function for fermionic replicas, i.e. for positive $n$, or by using bosonic replicas, i.e. for negative $n$.

An exact method that avoids difficulties of the replica trick is the supersymmetric method [16, 17, 18]. In that case the resolvent follows from the identity

$$
G(x)=\left.\frac{1}{N} \partial_{J}\left\langle\frac{\operatorname{det}(D+x+J)}{\operatorname{det}(D+x)}\right\rangle\right|_{J=0} .
$$

This method is not applicable, though, to cases where the partition function cannot be expressed as the average of a determinant, making it essential to understand the limitations of the replica limit. In this letter we will not discuss other alternatives to the replica method such as for example the dynamical method [19].

Both the generating functions for the replica trick and the supersymmetric method can be rewritten as a nonlinear $\sigma$-model. Generally, it is believed [20,21,5, 10, 12 that the replica limit can only be applied to asymptotic expansions such as the expansion in $1 / x$ of the resolvent. In [5] it was argued that the replica trick even fails to reproduce the oscillatory terms in the asymptotic expansions of correlation functions. Indeed, this is the case if one only takes into account the leading saddle point manifold. The oscillatory terms in the asymptotic expansion can be obtained from a subleading saddle point manifold [6] (see also [7.96). However, exact results could only be derived when the asymptotic expansion terminates [8, 11. In [8] this was related to the Duistermaat-Heckman localization property of the saddle point integrals.

A real breakthrough was recently made by Kanzieper 14. He succeeded to obtain exact nonperturbative results by means of the replica method. His approach was based on the property that the generating function for the resolvent or the two-point correlation function can be expressed in terms of a solution of a Painlevé equa- 
tion where the replica index only occurs as a parameter in its coefficients. The replica limit of the resolvent is then obtained from solution of this Painlevé equation for $n \rightarrow 0$. In particular, this allowed him to reproduce exact nonperturbative results with an asymptotic series that does not terminate. A first hint that it is possible to obtain such exact results surfaced in 11 where the leading logarithmic singularity of the small $x$ expansion of the resolvent was obtained from the replica trick.

In this letter we will analyze the replica trick for the two-point function of the Gaussian Unitary Ensemble (GUE) and for the microscopic limit of the resolvent of the chiral Unitary Ensemble (chUE) - the random matrix ensemble relevant for QCD 22].

The two-point function of the GUE can be expressed in terms of a generating function for $n$ fermionic replicas

$$
G(r)=-\lim _{n \rightarrow 0} \frac{1}{n^{2}} \frac{\partial^{2} Z_{n}(i r)}{\partial r^{2}}
$$

where the eigenvalue representation of the nonlinear $\sigma$ model for the generating function is given by [5]

$$
Z_{n}(i r)=n ! \int_{-1}^{1} \prod_{k=1}^{n} d \lambda_{k} e^{-i r \lambda_{k}} \Delta^{2}\left(\left\{\lambda_{k}\right\}\right) .
$$

Here, $\Delta\left(\left\{\lambda_{k}\right\}\right) \equiv \prod_{k<l}\left(\lambda_{k}-\lambda_{l}\right)$ is the Vandermonde determinant. For $n$ bosonic replicas, the two-point function is still given by (4) but with a generating function $Z_{-n}(r)$, that now can be expressed as an integral over a noncompact manifold [5],

$$
Z_{-n}(i r)=\frac{1}{n ![(n-1) !]^{2}} \int_{1}^{\infty} \prod_{k=1}^{n} d \lambda_{k} e^{i r \lambda_{k}} \Delta^{2}\left(\left\{\lambda_{k}\right\}\right)
$$

The sign of the exponent is determined by the sign of the infinitesimal increment of $\mathrm{ir}$. The fermionic generating function satisfies the Toda lattice equation [9]

$$
Z_{n}^{2}(i r) \partial_{r}^{2} \log Z_{n}(i r)=-\frac{n^{2}}{(n+1)^{2}} Z_{n+1}(i r) Z_{n-1}(i r)
$$

If we interpret the generating function for negative $n$ as the the bosonic generating function (6) this equation is valid for all integer values of $n$. In the replica limit, the l.h.s. of this equation is $n^{2}$ times the connected two-point function. On the r.h.s. we find from (5) and (6) that

$$
Z_{1}(i r) Z_{-1}(i r)=2 i \frac{\sin r}{r} \frac{e^{i r}}{r}
$$

which is the analytical result for the two-point function [5]! In [9] this result was obtained from the replica limit of the Painlevé V equation. The present method nicely explains the factorization of the two-point function into a compact and a non-compact contribution. In [8] it was argued that the replica limit reproduces the correct result in this case is because the asymptotic expansion in $1 / r$ terminates. We will now analyze the microscopic limit of the resolvent $G(x)$ of the chUE for which the asymptotic expansion in $1 / x$ does not terminate. Nevertheless, we will obtain the exact analytical result for $G(x)$ from the replica limit of a Toda lattice equation.

For topological quantum number $\nu$ the microscopic limit of the resolvent for the chUE is given by

$$
G(x)=x\left(K_{\nu}(x) I_{\nu}(x)+K_{\nu-1}(x) I_{\nu+1}(x)\right)+\frac{\nu}{x} .
$$

where $K_{\nu}(z)$ and $I_{\nu}(z)$ are modified Bessel functions. The most direct way to obtain this result is the supersymmetric method [23,24,15] where the appearance of the compact and noncompact integrals can be related to the symmetries of the underlying partition function [11]. However, it was first obtained in [25] by integrating the microscopic spectral density which can be easily derived from the orthogonal polynomial method 26,27,28. In [14 this result was obtained by solving the replica limit of the PIII Painlevé equation. In this approach, the appearance of noncompact integrals in the replica limit is a mystery. Here, we obtain the resolvent from the solution of the replica limit of the Toda lattice equation which relates the resolvent to both fermionic and bosonic partition functions. The noncompact integrals then arise from the bosonic partition function. This method is then generalized to the partially quenched case, where the replica limit of a generalized Toda lattice equation [29] relates the resolvent to a fermionic partition function and a partition function with both fermions and bosons.

The partition function of the chUE with $n$ fermionic replica flavors with mass $m$ and $N_{f}$ additional fermionic flavors with masses $m_{1}, \cdots, m_{N_{f}}$ is defined by

$$
Z_{\nu}^{\mathrm{chUE}}=\left\langle\operatorname{det}^{n}(D+m) \prod_{k=1}^{N_{f}} \operatorname{det}\left(D+m_{k}\right)\right\rangle .
$$

The Dirac operator is given by

$$
D \equiv\left(\begin{array}{cc}
0 & W \\
W^{\dagger} & 0
\end{array}\right)
$$

where $W$ is a rectangular $l \times(l+\nu)$ matrix so that $D$ has exactly $\nu$ zero eigenvalues. The average is over the probability distribution of the matrix elements of $W$. We will consider the microscopic limit of this partition function where the thermodynamic limit is taken for fixed $x \equiv m \Sigma N$ and $x_{k} \equiv m_{k} \Sigma N$. Here, $N=2 l+\nu$ and $\Sigma=\lim _{m \rightarrow 0, N \rightarrow \infty} \partial_{m} \log Z(m) / N$. In this case the partition function reduces to the unitary matrix integral 22$]$

$$
Z_{n, N_{f}}^{\nu}\left(x,\left\{x_{i}\right\}\right)=\frac{1}{C_{n}} \int_{U \in U\left(n+N_{f}\right)} d U \operatorname{det}^{\nu} U e^{\frac{1}{2} \operatorname{Tr}\left[M^{\dagger} U+M U^{\dagger}\right]}
$$

where $M=\operatorname{diag}\left(x, \cdots, x, x_{1}, \cdots, x_{N_{f}}\right)$ is the mass matrix and an additional normalization factor $C_{n}=\prod_{k=1}^{n}(k-1)$ ! 
has been included for later convenience. In the microscopic limit, this partition function coincides with the partition function of QCD 30,22]. The replica limit of the resolvent is then given by

$$
G\left(x,\left\{x_{i}\right\}\right)=\lim _{n \rightarrow 0} \frac{1}{n} \partial_{x} \log Z_{n, N_{f}}^{\nu}\left(x,\left\{x_{i}\right\}\right) .
$$

We first analyze the case of no additional massive flavors.

The quenched resolvent. In this case the partition function is given by

$$
Z_{n}^{\nu}(x)=\frac{1}{C_{n}} \int_{U \in U(n)} d U \operatorname{det}^{\nu} U e^{\frac{x}{2} \operatorname{Tr}\left[U+U^{\dagger}\right]} .
$$

The unitary matrix integrals are well-known 34, 35, 36, 37

$$
Z_{n}^{\nu}(x)=\frac{1}{C_{n}} \operatorname{det}\left[I_{\nu+j-k}(x)\right]_{j=1, \cdots, n, k=1, \cdots, n} .
$$

This expression is the $\tau$-function of an integrable hierarchy and satisfies the Toda lattice equation [31,29, 32,33.

$$
\left[x \partial_{x}\right]^{2} \log Z_{n}^{\nu}(x)=n x^{2} \frac{Z_{n+1}^{\nu}(x) Z_{n-1}^{\nu}(x)}{\left[Z_{n}^{\nu}(x)\right]^{2}} .
$$

The choice of the normalization constants $C_{n}$ is consistent with the large $x$ asymptotic behavior of the l.h.s. of (16) which is given by $n x$.

In the replica limit, $n \rightarrow 0$, the partition function can be expanded as

$$
Z_{n}^{\nu}(x)=1+n \sigma_{1}^{\nu}(x)+O\left(n^{2}\right) .
$$

In this limit, $Z_{-1}(x)$ appears in the r.h.s. of the Toda equation, which can be interpreted as the partition function with one bosonic replica. With normalization constant fixed by the large $x$ limit of (16) it is given by 11]

$$
Z_{-1}^{\nu}(x)=2 K_{\nu}(x) .
$$

For the replica limit of the partition function we thus obtain the differential equation

$$
n[x \partial x]^{2} \sigma_{1}^{\nu}(x)=2 n x^{2} I_{\nu}(x) K_{\nu}(x) .
$$

Using the identity

$\partial_{x} x^{2}\left[K_{\nu}(x) I_{\nu}(x)+K_{\nu-1}(x) I_{\nu+1}(x)\right]=2 x K_{\nu}(x) I_{\nu}(x)$,

this differential equation can be integrated resulting in

$\sigma_{1}^{\nu}(x)=\int_{0}^{x} d t\left[t\left(K_{\nu}(t) I_{\nu}(t)+K_{\nu-1}(t) I_{\nu+1}(t)\right)+\frac{\nu}{t}\right]$

in agreement with the known result (9) for the resolvent! The last term is a solution of the homogeneous differential equation with integration constants fixed by the small $x$ expansion of the partition function. We notice that the discontinuity of the resolvent arises through the bosonic integral $K_{\nu-1}(x)$ via the Toda lattice equation.
In this way, the analyticity in $x$ of the fermionic replica expression for the resolvent [8] is avoided. In [14], the expression for $\sigma_{1}^{\nu}$, was obtained from the solution of the replica limit of the PIII Painlevé equation. As a side remark, we notice that $K_{\nu}(x)$ is a solution of the PIII equation for $n \rightarrow-1$ satisfying the boundary conditions for $n=-1$. The PIII equation thus encodes the analyticity properties of the bosonic integral $K_{\nu}(x)$.

The partially quenched case. The partition function (12) satisfies the recursion relation [29]

$$
\begin{aligned}
& n x^{2} \frac{Z_{n+1, N_{f}}^{\nu}\left(x,\left\{x_{k}\right\}\right) Z_{n-1, N_{f}}^{\nu}\left(x,\left\{x_{k}\right\}\right)}{\left[Z_{n, N_{f}}^{\nu}\left(x,\left\{x_{k}\right\}\right)\right]^{2}} \\
= & \left.\sum_{k=0}^{N_{f}} x_{0} \partial_{x_{0}} x_{k} \partial_{x_{k}} \log Z_{n, N_{f}}^{\nu}\left(x_{0},\left\{x_{k}\right\}\right)\right|_{x_{0}=x} .
\end{aligned}
$$

This recursion relation can be derived from a special case of the Hirota equation. For degenerate masses $x_{k}$ the sum over the derivatives is only over the different masses. The normalization of the partition functions is again fixed by the large $x$ behavior of the r.h.s. at fixed $x_{k}$ which is given by $n x$.

Let us consider the replica limit of (22) for $N_{f}=1$. Then the numerator of the l.h.s. is the product of

$$
Z_{1,1}^{\nu}(x, y)=\frac{2}{y^{2}-x^{2}} \operatorname{det}\left|\begin{array}{cc}
I_{\nu}(x) & I_{\nu}(y) \\
x I_{\nu+1}(x) & y I_{\nu+1}(y)
\end{array}\right|,
$$

and the supersymmetric partition function 11

$$
Z_{-1,1}^{\nu}(x, y)=y K_{\nu}(x) I_{\nu+1}(y)+x K_{\nu+1}(x) I_{\nu}(y)
$$

with a bosonic mass equal to $x$ and a fermionic mass equal to $y$. In the replica limit, $Z_{n, 1}^{\nu}(x, y)$ reduces to the partition function for one flavor and we have

$$
Z_{n, 1}^{\nu}(x, y)=I_{\nu}(y)\left(1+n \sigma_{1}^{\nu}(x, y)+O\left(n^{2}\right)\right) .
$$

Both sides of the recursion relation are of order $n$. The quantity $\sigma_{1}^{\nu}$ thus satisfies the linear differential equation

$$
\left(x \partial_{x}+y \partial y\right) x \partial_{x} \sigma_{1}^{\nu}(x, y)=x^{2} \frac{Z_{-1,1}^{\nu}(x, y) Z_{1,1}^{\nu}(x, y)}{I_{\nu}(y)^{2}} .
$$

Using (20) and the identities

$$
\begin{aligned}
& \left(x \partial_{x}+y \partial_{y}\right)\left[y I_{\nu}(x) I_{\nu+1}(y)-x I_{\nu}(y) I_{\nu+1}(x)\right] \\
& =\left(y^{2}-x^{2}\right) I_{\nu}(x) I_{\nu}(y) \\
& I_{\nu}^{2}(y)\left(x \partial_{x}+y \partial_{y}\right) \frac{K_{\nu}(x)}{I_{\nu}(y)} \\
& =-\left[x K_{\nu+1}(x) I_{\nu}(y)+y K_{\nu}(x) I_{\nu+1}(y)\right]
\end{aligned}
$$

this equation can be integrated with solution given by

$$
\begin{aligned}
\sigma_{1}^{\nu}(x, y)= & \int_{0}^{x} t\left[I_{\nu}(t) K_{\nu}(t)+I_{\nu+1}(t) K_{\nu-1}(t)\right. \\
& \left.+\frac{\nu}{t^{2}}-\frac{K_{\nu}(t)}{I_{\nu}(y)} Z_{1,1}^{\nu}(t, y)\right] d t .
\end{aligned}
$$


The resolvent given by $\partial_{x} \sigma_{1}^{\nu}(x, y)$ agrees with earlier results obtained by the supersymmetric method 24].

The partially quenched resolvent can be worked out analogously for more flavors. For example we have obtained the resolvent for $N_{f}$ massless flavors and $\nu=0$ from the replica limit of the Toda lattice equation.

General Solutions. Away from the replica limit we can integrate the Toda lattice equation to determine the partition functions with arbitrary number of fermions and bosons but with some degenerate masses. Based on these results we conjecture the general form of the partition function for arbitrary masses. Up to a normalization constant we find for pure bosonic partition functions

$$
\frac{\operatorname{det}\left[x_{i}^{j-1} K_{\nu+j-1}\left(x_{i}\right)\right]_{i, j=1, . ., n}}{\prod_{j>i}\left(x_{j}^{2}-x_{i}^{2}\right)}
$$

which in the case of degenerate masses reduces to $Z_{-n, 0}(x)=\operatorname{det}\left[K_{\nu+j-i}(x)\right]$. This result agrees with the integral representation given in [11, 15]. The microscopic limit of the chUE partition function with $n$ bosons and $m$ fermions satisfies the generalized Toda lattice equation (22) continued to negative $n$. Iterating this equation we conjecture the following result for the partition function

$Z_{-n, m}^{\nu}\left(\left\{x_{i}\right\}\right)=\frac{\operatorname{det}\left[x_{i}^{j-1} \mathcal{J}_{\nu+j-1}\left(x_{i}\right)\right]_{i, j=1, . ., n+m}}{\prod_{j>i=1}^{n}\left(x_{j}^{2}-x_{i}^{2}\right) \prod_{j>i=n+1}^{n+m}\left(x_{j}^{2}-x_{i}^{2}\right)}$.

The symbol $\mathcal{J}_{\nu}\left(x_{i}\right) \equiv(-1)^{\nu} K_{\nu}\left(x_{i}\right)$ for $i=1, \ldots, n$ and $\mathcal{J}_{\nu}\left(x_{i}\right) \equiv I_{\nu}\left(x_{i}\right)$ for $i=n+1, \ldots, n+m$. The structure of this expression is in agreement with the general result obtained in [38]. For $\nu=0$ the result for $Z_{-n, m}^{\nu}$ was first derived by means of the supersymmetric method [39]. The partition function $(30)$ is relevant for the calculation of higher order spectral correlation functions [24,40].

Conclusions. The two-point function of the Gaussian Unitary Ensemble and the quenched and partially quenched resolvent for the microscopic limit of the chiral Unitary Ensemble have been obtained by solving the replica limit of a (generalized) Toda lattice equation. This method provides a direct analytical understanding of the appearance of compact and noncompact integrals in the expressions for the resolvent. It also explains the appearance of a discontinuity in the fermionic replica limit of the resolvent. The family of chUE partition functions with any number of fermions and bosons and with arbitrary masses and topological charge also satisfies the generalized Toda lattice equation. On this basis we have conjectured a form for this partition function that generalizes the result for zero topological charge.

Acknowledgements: We wish to thank P.H. Damgaard and M.R. Zirnbauer for useful discussion and suggestions. Note added: The expression for $Z_{-n, m}^{\nu}\left(\left\{x_{i}\right\}\right)$ in eq. (30) has now been verified [41] based on a result in 38.
[1] S.F. Edwards and P. Anderson, J. Phys. F5, 865 (1975).

[2] D. Sherrington and S. Kirkpatrick, Phys. Rev. Lett. 35, 1792 (1975).

[3] M. Mézard, G. Parisi and M.A. Virasoro, Spin Glass Theory and Beyond, World Scientific, Singapore 1987.

[4] M. Stephanov, Phys. Rev. Lett. 76, 4472 (1996).

[5] J.J.M. Verbaarschot and M.R. Zirnbauer, J. Phys. A18, 1093 (1985).

[6] A. Kamenev and M. Mézard, J. Phys. A, 32, 4373 (1999); Phys. Rev. B 60, 3944 (1999).

[7] I. Yurkevich and I. Lerner, Phys. Rev. B 60, 3955 (1999).

[8] M.R. Zirnbauer, cond-mat/9903338.

[9] E. Kanzieper, Nucl. Phys. B 596, 548 (2001).

[10] P.H. Damgaard and K. Splittorff,Nucl. Phys. B 572, 478 (2000); Phys. Rev. D 62, 054509 (2000);P.H. Damgaard, Phys. Lett. B 476, 465 (2000).

[11] D. Dalmazi and J.J.M. Verbaarschot, Nucl. Phys. B 592, 419 (2001).

[12] G. Akemann et al., Nucl. Phys. B 601, 77 (2001).

[13] S.M. Nishigaki and A. Kamenev, J. Phys. A: Math. Gen. 35, 4571 (2002).

[14] E. Kanzieper, cond-mat/0207745.

[15] Y. V. Fyodorov, Nucl. Phys. B 621, 643 (2002).

[16] E. Brézin, Lect. Notes Phys. 216, 113 (1980).

[17] K.B. Efetov, Adv. Phys. 32, 53 (1983).

[18] J.J.M. Verbaarschot, H.A. Weidenmüller, and M.R. Zirnbauer, Phys. Rep. 129, 367 (1985).

[19] A. Altland and A. Kamenev, Phys. Rev. Lett. 85, 5615 (2000).

[20] S.F. Edwards and R.C. Jones, J. Phys. A 9, 1595 (1976).

[21] J.J.M. Verbaarschot and M.R. Zirnbauer, Ann. Phys. 158, 78 (1984).

[22] E.V. Shuryak and J.J.M. Verbaarschot, Nucl. Phys. A 560, 306 (1993); J.J.M. Verbaarschot, Phys. Rev. Lett. 72, 2531 (1994).

[23] J.C. Osborn et al., Nucl. Phys. B 540, 317 (1999).

[24] P.H. Damgaard et al., Nucl. Phys. B 547, 305 (1999).

[25] J.J.M. Verbaarschot, Phys. Lett. B 368, 137 (1996).

[26] P.J. Forrester, Nucl. Phys. B 402, 709 (1993).

[27] J. Verbaarschot and I. Zahed, Phys. Rev. Lett. 70, 3852 (1993); J. Verbaarschot, Nucl. Phys. B 427, 534 (1994).

[28] G. Akemann et al., Nucl. Phys. B 487[FS], 721 (1997).

[29] S. Kharchev et al., Nucl. Phys. B 380, 181 (1992).

[30] H. Leutwyler, A. Smilga, Phys. Rev. D 46, 5607 (1992).

[31] K. Okamoto, Funkcialaj Ekvacioj 30, 305, (1987).

[32] P.J. Forrester and N.S. Witte, math-ph/0201051.

[33] G. Akemann and P.H. Damgaard, Nucl. Phys. B 576, 597 (2000).

[34] R.C. Brower, P. Rossi and C-I. Tan, Nucl. Phys. B 190, 699 (1981); R.C. Brower and M. Nauenberg, Nucl. Phys. B 180, 221 (1981).

[35] A.D. Jackson et al., Phys. Lett. B 387, 355 (1996).

[36] T. Guhr and T. Wettig, Nucl. Phys. B 506, 589 (1997).

[37] A.B. Balantekin, Phys. Rev. D 62, 085017 (2000).

[38] Y.V. Fyodorov and E. Strahov, math-ph/0204051.

[39] Y.V. Fyodorov and E. Strahov, hep-th/0205215.

[40] B. Seif et al., Nucl. Phys. B 548, 475 (1999).

[41] Y.V. Fyodorov and G. Akemann, cond-mat/0210647. 